\title{
PROPUESTA DE UN MODELO PARA EL ANÁLISIS DE LA IMPLEMENTACIÓN DE INICIATIVAS ESTATALES ORIENTADAS AL MEJORAMIENTO EDUCATIVO $^{1}$
}

\section{Luis Felipe De la Vega}

\section{RESUMEN}

El presente artículo da cuenta de una investigación acerca de la implementación de iniciativas estatales del sistema educativo chileno que buscan aportar al mejoramiento educativo.

Considerando un marco conceptual propio del estudio de la implementación de políticas públicas en general y de las referidas a la educación en particular, se llevó a cabo una revisión sistemática de cincuenta y dos estudios realizados o solicitados por instituciones estatales, respecto de iniciativas que buscaban aportar al mejoramiento educativo.

A partir de esta revisión, se identificaron factores relacionados con la implementación de las iniciativas que afectan su aporte al logro de sus propósitos; también, factores vinculados a la interpretación de las iniciativas que realizan los diversos actores implicados y con las respuestas que las iniciativas suscitan en ellos. Finalmente, se buscó reconocer aquellos factores que inciden en las adaptaciones que las iniciativas experimentan en el proceso de su implementación.

A partir de este análisis, se propone un modelo para analizar la implementación de las iniciativas estatales, que permite identificar los factores que deben ser considerados con el fin de potenciar el aporte de estas al mejoramiento educativo.

Conceptos clave: políticas educativas, mejoramiento educativo, implementación de políticas, políticas públicas.

\section{PROPOSAL OF A MODEL FOR THE ANALYSIS OF THE IMPLEMENTATION OF STATE INITIATIVES IN THE AREA OF EDUCATION IMPROVEMENT}

\section{ABSTRACT}

This article presents research on the implementation of state initiatives for the Chilean education system, that seek to contribute to the improvement of education.

Considering a conceptual framework unique to the study of the implementation of public policy, in general, and those relating to education, in particular, a systematic review was carried out of fifty-two studies that were conducted or requested by state institutions, regarding initiatives that sought to contribute to the improvement of education. This review identified factors related to the achievement of the initiatives' intended objectives, factors associated with the interpretations of

1 Proyecto apoyado financieramente por CNED/ Convocatoria 2018.

2 Universidad de Chile, Santiago,Chile. Contacto: luis.delavega@uchile.cl 
78 PROPUESTA DE UN MODELO PARA EL ANÁLISIS DE LA IMPLEMENTACIÓN DE INICIATIVAS ESTATALES ORIENTADAS AL MEJORAMIENTO EDUCATIVO - L. De la Vega

said initiatives made by the diverse actors and how they choose to act in response, and factors that influence the modifications made to the initiatives during the implementation process.

Through this analysis, a model is proposed to analyze the implementation of the state initiatives, that permits the identification of factors that should be considered, with the goal of strengthening their contribution to the improvement of education.

Key concepts: education policies, education improvement, policy implementation, public policy 


\section{Introducción}

En las últimas décadas, los sistemas educativos han ido definiendo al mejoramiento educativo como uno de sus propósitos fundamentales (Mourshed, Chijioke \& Barber, 2010). Este concepto da cuenta del desarrollo de cambios educativos en diferentes niveles y áreas, pero sobre todo en la transformación positiva de la formación y aprendizaje de los estudiantes (Bellei, Valenzuela, Vanni y Contreras, 2014; Elmore, 2010; Fullan, 2002; Rincón-Gallardo, 2016). De esta forma, las innovaciones, los procesos organizacionales, las orientaciones simbólicas y el desarrollo de capacidades involucrados en el mejoramiento educativo, solo pueden considerarse eficaces si inciden positivamente en el proceso de enseñanza y aprendizaje (Elmore, 2010; Fullan; 2011; Hopkins; 2008; Levin; 2009).

Una de las características más relevantes del mejoramiento educativo es su naturaleza procesual, lo que ha propiciado que se preste especial atención a las formas en que se realiza el cambio en educación y no únicamente a sus resultados finales. Anderson (2012) observa que el mejoramiento educativo puede seguir distintas vías y que su desarrollo no necesariamente es lineal. Pese a ello, de acuerdo con Hallinger y Heck (2011), sí es necesario que exista una ruta o camino hacia el mejoramiento que se oriente hacia la sustentabilidad del proceso (Fullan, 2016).

Dada su relevancia en los procesos escolares, las iniciativas estatales en materia educativa pueden ser un importante factor para propiciar el mejoramiento educativo, en la medida en que se orienten a afectar al sistema educativo y a las instituciones o actores que forman parte de este, ya sea para promover cambios o para incidir en que estos se mantengan o profundicen.

¿De qué forma se implementan las iniciativas estatales para aportar al mejoramiento educativo? El presente artículo indaga en este fenómeno, preguntándose por los factores y elementos que intervienen en la implementación de estas iniciativas, de manera de recoger aprendizajes que aporten a comprender este fenómeno y a mejorar su eficacia. Cabe señalar que, si bien existen múltiples investigaciones respecto de políticas públicas en educación en 
Chile, no se advierte un desarrollo de robustos cuerpos teóricos o metodológicos, o de metaanálisis, específicamente orientados a su estudio.

\section{Marco conceptual}

Diferentes aprendizajes y hallazgos han ayudado a profundizar en la comprensión de la implementación de las iniciativas estatales en materia de políticas educativas y su aporte a la mejora escolar. Gallucci, Knapp, Markholt y Ort (2006), y Anderson (2012), consideran que las iniciativas que buscan favorecer la mejora deben combinar estrategias en los niveles central y local, para adaptar los requerimientos del nivel central a la realidad del establecimiento educacional.

Chrispeels y Harris (2006) señalan que estas iniciativas deben disponer de estrategias que consideren un conjunto de interrogantes, tales como qué medidas de mejoramiento favorecen cambios positivos para el sistema y el nivel local; cómo compatibilizar el desarrollo del trabajo autónomo de los docentes con la necesidad de fortalecer el aprendizaje colectivo, o cómo hacer escalar reformas, combinando capacidades sistémicas y locales.

Otro ámbito del que han emergido hallazgos referidos a la implementación de las iniciativas estatales orientadas a la mejora escolar, alude a las respuestas a ellas de parte de los actores. Se ha ido constatando que los actores involucrados no necesariamente se alinean con las políticas, sino que las reinterpretan y responden a ellas en función de sus características, condiciones, intereses y posibilidades, en relación al contexto y las instituciones en las que operan. De esta forma, habrá respuestas más o menos cercanas a las expectativas de la iniciativa (Day $\&$ Gu, 2018; Bryan, Ko $\&$ Walker, 2018; Gu, Sammons \& Chen, 2018) y es altamente probable que existan brechas importantes entre el diseño y la implementación (Dedering \& Miller, 2011).

Las explicaciones más comunes respecto de las dificultades en la respuesta técnica de los actores educativos a estas iniciativas es que 
su implementación no es suficiente para movilizar transformaciones en las capacidades de los actores. Así lo muestran investigaciones como las de Strunk, Marsh, Hashim y Bush-Mecenas (2016) y Strunk, Marsh, Bush-Mecenas y Duque (2016), que dan cuenta de posibles choques entre las lógicas de las iniciativas estatales, las organizaciones y los actores, y observan que las respuestas de los actores también pueden variar en el tiempo.

La perspectiva de la mejora escolar ha establecido ciertas definiciones o proposiciones respecto de la manera en que debieran conducirse las iniciativas estatales que se orientan al mejoramiento educativo. Este tipo de definiciones ha tenido alta influencia en los sistemas educativos, lo que se refleja en el uso cotidiano que adquieren términos como "núcleo pedagógico", "liderazgo sistémico" o "desarrollo de capacidades" (Hopkins, 2017).

Levin (2007) y Fullan (2010) relevan la condición sistémica del cambio, al decir que hay un conjunto de elementos que deben combinarse positivamente entre sí para avanzar en el mejoramiento educativo. Levin propone que las claves para generar estos procesos sinérgicos de mejoramiento son: a) respeto por el staff y el profesionalismo de los docentes; b) comprehensividad (considerar amplias expectativas de logro); c) alineamiento a través de la colaboración; d) mayores recursos, utilizados focalizadamente; e) accountability menos castigador, y f) liderazgo político.

Barber (2008) y Fullan (2011) indican que los sistemas educativos deben escoger y desarrollar los "conductores" adecuados de reformas que redunden en mejoras de los aprendizajes de los estudiantes. Fullan menciona que muchos sistemas educativos escogen mal los "conductores", al considerar como tales, por ejemplo, un accountability relacionado con mediciones estandarizadas, el foco en las capacidades individuales de los docentes (en desmedro de las colectivas), el uso de tecnologías como la solución a los problemas y/o la implementación de estrategias fragmentadas y no sistémicas.

Desde una posición más crítica, Hargreaves (2010) señala que las reformas orientadas a la mejora debieran ocurrir a partir 
del trabajo de los docentes entre sí, lo que implica que las escuelas mejorarán realmente solo cuando se conecten con otras y se generen comunidades de aprendizaje profesionales (Hargreaves \& Fullan, 2012; Hargreaves \& O'Connor, 2018).

En la misma línea, Harris (2012) indica que muchas reformas no generan mejoramiento educativo porque se sostienen en alguna de las siguientes falacias: a) el mejoramiento educativo es rápido y sencillo; b) se requiere alto reemplazo del personal, de líderes y de docentes, y c) la estandarización, prescripción y accountability llevarán definitivamente al mejoramiento. De acuerdo con Harris, Jones y Adams (2016), las iniciativas estatales en educación debieran potenciar que los profesionales de las instituciones educativas identifiquen sus propias prioridades de mejora y establezcan objetivos comunes y cuantificables para el progreso.

Hopkins (2013 y 2017) añade que uno de los grandes errores de la conducción de las reformas escolares, que ha llevado a múltiples fracasos, es la aplicación de soluciones empaquetadas en diferentes contextos, impuestas en una lógica top down. En vez de ello, Hopkins señala que se deben desarrollar teorías de acción basadas en la realidad de los hechos.

Schleicher (2016) agrega que, para conducir las reformas, los "hacedores de política" deben buscar generar consensos e incorporar a los actores involucrados tanto en el diseño como en la implementación de las políticas, brindando seguridad de que se les entregará herramientas para enfrentar el cambio esperado.

Los sistemas de aseguramiento de la calidad — como el chileno- esperan incidir de forma determinante en el mejoramiento educativo. Sin embargo, dada la complejidad de los sistemas educativos, es común y esperable que los resultados de la implementación de las iniciativas estatales tengan diferencias en distintos escenarios, y que en ello incida un conjunto de factores. En ese sentido, la implementación de estas iniciativas requiere contar con evidencia que permita tomar decisiones informadas para potenciar sus resultados, haciendo ajustes, modificándolas o agregando otras acciones complementarias (Gill, Coffee \& Hallgren, 2014). 
La literatura chilena no ha sido prolífica en analizar el concepto de "implementación" propiamente tal. Si bien diferentes investigaciones han estudiado numerosas políticas educativas, no lo han hecho desde la reflexión conceptual respecto de este término. Pese a ello, en los últimos años se verifican propuestas analíticas referidas a este objeto de estudio (Aziz, 2018). Por esta razón, parece relevante contar con una aproximación teórica a este fenómeno.

La implementación es una etapa que abarca gran parte de la vida de una iniciativa estatal en cualquier ámbito. En esta etapa se inicia la puesta en marcha de la iniciativa diseñada, y se produce el encuentro de las ideas — expresadas en diseños y normativas — con la realidad. Es el espacio en que se comprueban las hipótesis o lógicas de cambio o transformación de la iniciativa en cuestión (Durlak \& Dupré, 2008). Para Parsons (2007), la implementación es un proceso en que se aplican ideas políticas a través de acciones racionalmente planificadas y en que, además, se observa la multiplicidad de eventos, intereses y actores que intervienen, y que pueden modificar la planificación.

Los estudios sobre implementación de políticas públicas buscan reconocer lo que ocurre con el escenario, los hechos y las partes involucradas durante este proceso. Históricamente ha existido una tensión respecto de si debiera priorizarse la identificación de variables centrales para la gestión exitosa de la política o programa, o si, más bien, debiera enfatizarse la comprensión de los fenómenos que emergen desde la implementación. Esta tensión es la que acompaña la discusión referida a los enfoques de análisis conocidos como top down vs. los bottom up (Pülzl, 2007; Revuelta, 2008). Los primeros priorizan el análisis racionalizado del proceso de desarrollo de iniciativas estatales (Van Meter, 1975; Bardach, 1976). Los segundos buscan comprender los fenómenos que ocurren en la implementación, partiendo "desde abajo hacia arriba" y no preguntándose necesariamente por el apego al diseño inicial (Elmore, 2003). Con el transcurrir de la historia de estos estudios ha ido haciéndose evidente la necesidad de integrar ambas perspectivas (Barrett, 2004; De Leon \& Varda, 2009; Sabatier, 2003). 
Además del avance en el debate y los hallazgos respecto del estudio de la implementación de las iniciativas estatales o políticas públicas en general, el análisis de la implementación también ha tenido lugar en el ámbito educativo. A continuación se presentan tres perspectivas desde las cuales se ha abordado este análisis ${ }^{3}$.

\section{Perspectiva con foco en las instituciones que implementan}

Una primera perspectiva teórica pone el foco en el sistema educativo y las instituciones y organizaciones que lo administran. Éstas buscan comprender la implementación para definir lineamientos que potencien el diseño y la ejecución de las iniciativas del sistema educativo. Coburn (2003) utiliza el concepto de "escala" para dar cuenta de las necesidades y tensiones que deben considerar las políticas educativas, con el fin de impactar positivamente en un conjunto amplio y diverso de instituciones y actores. El escalamiento de una iniciativa a un nivel propio del sistema educativo requeriría considerar cuatro dimensiones: a) profundidad, vale decir, el grado en que se producen transformaciones significativas y profundas en las prácticas educativas; b) sustentabilidad, o la generación o reforzamiento de las condiciones y recursos para apoyar la consolidación y expansión de las reformas en el tiempo; c) difusión, es decir, el grado en que la reforma expande sus prácticas hacia nuevos sitios o grupos, y d) cambio de propiedad de la reforma, entendido como la manera en que el conocimiento y la agencia para sostener la reforma se transfieren al nivel local.

El desafío de una política, en su escalamiento, es alcanzar mayor envergadura e involucrar a más procesos y actores sin afectar al núcleo pedagógico (Rincón-Gallardo, 2014; Rincón-Gallardo \& Fleisch, 2016).

Kit Looi y Woon Teh (2015) observan avances en las investigaciones realizadas con este enfoque, que han permitido evaluar

3 Es importante indicar que también existen otras perspectivas más críticas (Apple, 2011; Ball, 2016; Lipman, 2013), las que no se consideraron en esta revisión, por considerar que enfocan su análisis más en el diseño que en la implementación de políticas o reformas educativas. 
qué tipo de capacidades personales e institucionales se requieren para poner en práctica determinadas innovaciones y enfrentar de mejor forma los cambios, integrando visiones que se orientan a armonizar la optimización de la búsqueda de fidelidad en la implementación con las oportunidades y desafíos que presentan los contextos locales.

Como puede observarse, el foco de estas perspectivas está puesto en aquello que se implementa, identificando variables que afectan en el despliegue adecuado de las iniciativas. Esta lógica guarda similitudes, en términos de visión, con la llamada "ciencia de la implementación", enfoque mayormente utilizado para el análisis de intervenciones y programas, que busca identificar, medir y valorar aquellos elementos que inciden en una implementación fiel al diseño, y que además logre los objetivos que se esperan de una determinada iniciativa. De esta forma, se busca que los programas se desarrollen sobre la base de evidencia empírica rigurosa (Nilsen, 2015; Sanetti $\&$ Collier-Meek, 2015).

Perspectiva con foco en la interpretación de los procesos de implementación

Una segunda perspectiva de estudios de la implementación ha priorizado la interpretación y reinterpretación de las iniciativas estatales por parte de los actores educativos. Spillane (2002) indica que la comprensión de la implementación de una iniciativa estatal en materia de política educativa no pasa solo por la revisión organizacional de su diseño y gestión, sino que también requiere entender la visión subjetiva e intersubjetiva de los involucrados, y el vínculo entre esta y la acción social.

Esta perspectiva, definida como de "dar sentido" (sensemaking, en inglés), sitúa su análisis en el terreno en que se implementa la política, el espacio en que se construye socialmente. Para ello, enfatiza la mirada sobre quienes participan del proceso de implementación, la que se vincula con el contexto específico en que estos actores se sitúan.

Lester, Lochmiller y Gabriel (2017), y Lochmiller y Hedges (2017) afirman que la última década ha estado marcada por una alta cantidad de investigaciones que consideran esta perspectiva. 
Sus resultados permiten identificar el efecto que tiene la respuesta o las posiciones que adoptan los actores respecto de una iniciativa estatal en su implementación; el alineamiento entre una determinada iniciativa y las perspectivas y necesidades en el sistema local o de las escuelas; la influencia de las iniciativas en las perspectivas y las prácticas de los actores involucrados, o el rol estratégico que pueden adquirir algunos de estos para el éxito de una iniciativa.

\section{Perspectivas con foco en la coconstrucción.}

Una tercera perspectiva para el estudio de la implementación de las iniciativas estatales en materia de políticas educativas aspira a una integración entre las que enfatizan la mirada de la política y las organizaciones, con las que priorizan el estudio de los sujetos y escenarios de implementación.

Datnow (2000, 2005; Datnow \& Park, 2009) propone una aproximación a la implementación de políticas educativas como "coconstrucción", esto es, como el estudio relacional de las políticas, mediadas por el contexto e influidas por el vínculo que establecen con los actores involucrados. Esta perspectiva asume que la implementación genera efectos y transformaciones en múltiples direcciones, incluida la misma política, e indica que es necesario situar el análisis en contextos específicos de implementación para comprender los efectos y resultados de una política educativa. Por esta razón, es necesario considerar los escenarios específicos de implementación, asumiendo una lógica relacional (entre los actores, las instituciones y las organizaciones involucradas) para comprender los efectos y resultados de la puesta en marcha de una intervención.

Schleicher (2016) releva lo impredecible que puede llegar a ser la implementación, por la alta cantidad de factores y los variados intereses y posiciones de poder de los actores que allí intervienen, aspectos que pueden convertirse en facilitadores u obstaculizadores. El éxito de la implementación requiere considerar las capacidades disponibles en los diferentes niveles, para responder a sus roles y responsabilidades, los mecanismos de coordinación y articulación (gobernanza) entre las instituciones y organismos involucrados, y la 
disponibilidad de información relevante para la toma de decisiones en los diferentes niveles del sistema.

En este marco, Coburn, Hill y Spillane (2016) observan que el estudio de la implementación debiera avanzar en la comprensión de la complejidad de las políticas, entendiendo, por ejemplo, que estas van mutando en el tiempo y que sus resultados finales no se obtienen en el corto plazo.

Considerando todos estos elementos, y a partir del análisis de reportes de investigaciones y evaluaciones de la ejecución de normas, políticas, programas y proyectos, este trabajo propondrá un modelo para el análisis de la implementación de iniciativas estatales que buscan propiciar el mejoramiento del sistema educativo y/o de los establecimientos educacionales. Este modelo permite identificar claves que contribuyen al logro de los objetivos de los procesos de implementación de las iniciativas estatales orientadas al mejoramiento educativo.

\section{Metodología}

La presente investigación consideró el desarrollo de una "revisión sistemática" de información. Higgins y Green (2011) definen esta metodología como la identificación, análisis y síntesis de la evidencia respecto de un problema de estudio.

En particular, se buscó sistematizar y analizar un corpus de estudios específicamente referidos a la implementación de políticas, programas o proyectos educativos liderados por el Estado - los que se denominaron genéricamente como "iniciativas estatales"-, que buscan aportar al mejoramiento educativo.

Se consideraron aquellos estudios desarrollados o encargados por las propias instituciones del Estado, que analizan iniciativas ejecutadas por instituciones del Sistema de Aseguramiento de la Calidad, cuyo objetivo pudiera vincularse a la expectativa de mejoras en el núcleo pedagógico y/o en variables que podrían impactar en él (Elmore, 2010). Así, se incluyeron investigaciones sobre iniciativas 
orientadas a la mejora de las condiciones, la labor o los resultados de los docentes y la enseñanza, el curriculum y los aprendizajes, así como las que apuntaban a la mejora de la gestión de las instituciones escolares ${ }^{4}$.

De manera más específica, y siguiendo las definiciones de criterios propuestas por Botella y Zamora (2017), se incluyeron: 1) estudios que analizaran iniciativas orientadas a los ámbitos ya mencionados, desarrolladas por el Mineduc (incluyendo Fonide), la Agencia de Calidad de la Educación, la Superintendencia de Educación y el Programa de Evaluación de Programas Gubernamentales de DIPRES; 2) estudios finalizados entre 2014 y 2018, y 3) estudios que abordaran el análisis de la implementación de las iniciativas. Esto último implica que se excluyó a las investigaciones cuyo objeto de estudio fuera el diseño, los resultados, efectos o impactos de iniciativas estatales orientadas a la mejora educativa.

\section{Estrategias de búsqueda}

La primera etapa de la búsqueda consideró la totalidad de los estudios desarrollados o solicitados por las instituciones mencionadas que cumplieran con criterios de inclusión generales (de instituciones y años considerados). Se identificaron a 248 informes disponibles, a los que se accedió desde el sitio web de las instituciones o de su entrega como respuesta a una solicitud hecha por el investigador responsable a través de portales de transparencia.

De ese universo, y mediante la revisión de sus resúmenes, en la segunda etapa de la búsqueda se seleccionaron los estudios que cumplían con los criterios de inclusión referidos a contenidos.

La tabla 1 identifica la totalidad de estudios considerados en las dos etapas de búsqueda y el número de aquellos que fueron finalmente considerados en la investigación ${ }^{5}$.

4 Se excluyeron estudios de CNED, para garantizar la ausencia de conflicto de intereses en relación con el financiamiento que obtuvo esta investigación.

5 Se presenta en un anexo la lista de informes que fueron finalmente considerados para esta investigación. 
Tabla 1.

Estudios identificados y seleccionados para la investigación

\begin{tabular}{|c|c|c|}
\hline Institución & $\begin{array}{c}N^{\circ} \text { de documentos } \\
\text { fase } 1\end{array}$ & $\begin{array}{l}N^{\circ} \text { estudios } \\
\text { fase } 2\end{array}$ \\
\hline Mineduc (Incluye Fonide) & 178 & 30 \\
\hline Agencia de Calidad de la Educación & 43 & 11 \\
\hline Superintendencia de Educación ${ }^{6}$ & 2 & 0 \\
\hline EPG Dipres & 25 & 10 \\
\hline TOTAL & 248 & 52 \\
\hline
\end{tabular}

Fuente: Elaboración propia.

\section{Mecanismos de codificación y análisis}

De los informes seleccionados, las unidades de análisis fueron las secciones "Resultados", "Conclusiones" y "Recomendaciones", las que fueron objeto de análisis de contenido (Valles, 2000).

Utilizando antecedentes presentados en la revisión de la literatura sobre la implementación de políticas en general y de políticas educativas en particular, se identificaron aquellos elementos fundamentales para comprender a cabalidad estos procesos. Estos se organizaron en las siguientes categorías y se utilizaron para codificar los contenidos asociados a la implementación de las iniciativas estatales presentados en los informes considerados en esta investigación: a) tipo de iniciativa; b) involucrados en las iniciativas y vínculos entre ellos; c) principales procesos de implementación, características y procesos de los organismos ejecutores; d) calidad del diseño de la iniciativa; e) fortalezas del diseño que se evidencian en la implementación; f) debilidades del diseño que se evidencian en la implementación; g) fortalezas de la implementación; h) debilidades de la implementación; i) interpretaciones que se hacen de la iniciativa; $\mathrm{j}$ ) adaptaciones realizadas a la iniciativa durante la implementación.

Para realizar esta tarea, se seleccionaron párrafos textuales de los informes, los que fueron disponiéndose en las categorías recién mencionadas. Luego, estos textos fueron agrupados en clasificaciones

6 El bajo número de estudios considerados y seleccionados de esta institución se debe a que no se encontró estudios disponibles que cumplieran con los criterios de inclusión utilizados. 
estructuradas para propiciar el análisis comparativo entre informes (Cáceres, 2003).

A partir de la visión comparada de los diferentes informes, se organizó un análisis que da cuenta del proceso de implementación de las iniciativas, de los factores que inciden en él y de su aporte al mejoramiento educativo. Con ello, se elaboró una primera propuesta de modelo para el análisis y la evaluación de la implementación de iniciativas estatales que buscan favorecer el mejoramiento educativo. Se entiende como "modelo explicativo" una organización sistémica y sintética de los factores que tienen incidencia en el proceso de implementación de las iniciativas estatales y de la forma en que estos -y los elementos que los conforman— se relacionan entre sí.

Esta propuesta fue presentada, en entrevistas individuales, a representantes de equipos de investigación que se desempeñan en estudios de políticas educativas. Se llevaron a cabo ocho entrevistas con estos actores: cuatro de ellas a representantes de los organismos públicos asociados a los reportes estudiados (Ministerio de Educación, Agencia de Calidad de la Educación, Dirección de Presupuestos) y cuatro a representantes de instituciones ejecutoras de estos estudios (personas naturales, organismos consultores o instituciones de educación superior).

El objetivo de estas entrevistas fue validar el modelo realizado y enriquecerlo sobre la base de la perspectiva de estos expertos. Para ello, en primer lugar, se consultó a los entrevistados por los factores que consideraban más relevantes para comprender la implementación de las iniciativas desarrolladas por su institución o las que han estudiado. Luego, se hizo una presentación de los hallazgos de la investigación para, posteriormente, presentar el modelo explicativo. A partir de allí, los entrevistados entregaron comentarios y sugerencias para fortalecer el modelo.

Los resultados del análisis de las entrevistas favorecieron el desarrollo de una segunda versión del modelo, que se presentará al final del artículo. 


\section{Resultados}

\section{Tipos de iniciativa}

En las primeras fases del análisis de los estudios sobre iniciativas estatales orientadas a la mejora educativa, luego del registro y sistematización de la información en la base de datos — denominado preanálisis por Bardin (1996)—, se observó que los procesos de implementación tenían características asociadas al tipo de iniciativa que estaba siendo analizada. Por esta razón, el primer ejercicio para organizar la información sistematizada fue elaborar tipologías de las iniciativas. Se reconocieron seis tipos y, en una de ellas, dos subtipos. A continuación, se presentan sus características principales (tabla 2). 
Tabla 2.

Identificación de tipologías de iniciativas analizadas

\begin{tabular}{|c|c|c|}
\hline Tipo & Características generales del diseño & $\begin{array}{c}\mathrm{N}^{\circ} \text { de estudios } \\
\text { analizados }\end{array}$ \\
\hline $\begin{array}{l}\text { 1. Desarrollo de } \\
\text { capacidades }\end{array}$ & $\begin{array}{l}\text { Dispositivos para el mejoramiento de las } \\
\text { capacidades de las instituciones y actores } \\
\text { educativos, a través de la puesta en práctica de } \\
\text { un diseño que debe implementarse con el público } \\
\text { objetivo. La mayor parte son implementados por } \\
\text { un ejecutor intermediario. }\end{array}$ & 8 \\
\hline $\begin{array}{l}\text { 2. Generación } \\
\text { de condiciones } \\
\text { y apoyos a los } \\
\text { estudiantes }\end{array}$ & $\begin{array}{l}\text { Acompañamiento y desarrollo de condiciones en } \\
\text { estudiantes que, por razones socioeconómicas, no } \\
\text { desarrollan una experiencia educativa regular y/o } \\
\text { no logran objetivos o metas de aprendizaje en } \\
\text { igualdad de condiciones. Puede ser ejecutado por } \\
\text { una o más instituciones. }\end{array}$ & 6 \\
\hline $\begin{array}{l}\text { 3. Entrega de } \\
\text { apoyos para } \\
\text { la práctica } \\
\text { pedagógica }\end{array}$ & $\begin{array}{l}\text { Apoyo directo a la mejora de los procesos o } \\
\text { la gestión pedagógica, a través de la entrega } \\
\text { de recursos diseñados desde el nivel central. } \\
\text { Algunas incluyen otros componentes como } \\
\text { acompañamiento, orientaciones o definición de } \\
\text { roles internos. }\end{array}$ & 7 \\
\hline $\begin{array}{l}\text { 4. Regulación } \\
\text { de procesos } \\
\text { y prácticas } \\
\text { educativas }\end{array}$ & $\begin{array}{l}\text { Reglamentación (normativa, decreto, ley u } \\
\text { otra) sobre cómo debe operar determinado } \\
\text { procedimiento, proceso o práctica en el sistema } \\
\text { educativo, definiendo responsabilidades, tareas, } \\
\text { prácticas o sentidos. }\end{array}$ & 7 \\
\hline $\begin{array}{l}\text { 5. Incentivo } \\
\text { financiero y } \\
\text { generación de } \\
\text { condiciones }\end{array}$ & $\begin{array}{l}\text { Transferencia de recursos monetarios para } \\
\text { favorecer la implementación de procesos o } \\
\text { trayectorias educativas. Los destinatarios pueden } \\
\text { ser actores educativos (mayoritariamente } \\
\text { estudiantes) o instituciones educativas. Los } \\
\text { recursos se entregan luego de procesos de } \\
\text { postulación o priorización. }\end{array}$ & 8 \\
\hline $\begin{array}{l}6.1 \text { Mejora } \\
\text { sistémica del } \\
\text { funcionamiento } \\
\text { y resultados de la } \\
\text { educación }\end{array}$ & $\begin{array}{l}\text { Definiciones sobre qué se espera del } \\
\text { funcionamiento de los establecimientos en } \\
\text { determinados ámbitos de función o gestión, o } \\
\text { cómo estos elementos debieran combinarse en } \\
\text { conjunto. En algunos casos incluyen procesos de } \\
\text { supervisión, evaluación o acompañamiento de } \\
\text { su funcionamiento o sus resultados, o recursos } \\
\text { financieros. }\end{array}$ & 12 \\
\hline $\begin{array}{l}\text { 6.2. Mejora } \\
\text { sistémica a través } \\
\text { de accountability }\end{array}$ & $\begin{array}{l}\text { Políticas orientadas a la mejora sistémica a } \\
\text { través de mecanismos de rendición de cuentas, } \\
\text { específicamente performativos. }\end{array}$ & 3 \\
\hline
\end{tabular}

Fuente: Elaboración propia. 


\section{Características del diseño de las iniciativas}

Se identificaron particularidades en las características del diseño de las iniciativas. En primer lugar, independiente de su tipo, el nivel central del Estado es el que establece las definiciones fundamentales, los contenidos, las responsabilidades y los procesos asociados.

En función de sus contenidos, los siete tipos de iniciativas identificadas pueden ordenarse en un continuo en relación con su foco, desde el desarrollo de capacidades de instituciones y actores hacia la rendición de cuentas al Estado. Este continuo da cuenta de qué tan próxima está la iniciativa respecto del núcleo pedagógico, y de hacia dónde se dirige la acción fundamental de esta (hacia actores educativos o hacia el Estado).

Los involucrados en las diferentes iniciativas estatales consideran al menos el nivel central y las instituciones educativas o actores al interior de ellas. En algunos casos, contemplan instituciones o actores que actúan como intermediarios o ejecutores, los que pueden o no ser funcionarios públicos o miembros del nivel intermedio (sostenedor). Otro patrón común, en términos de involucrados, es que, así como el nivel central tiene un gran protagonismo en el diseño y control de los procesos, los actores educativos cumplen más bien un rol de beneficiarios o de cumplidores de obligaciones que ya han sido definidas sin su participación.

Los vínculos generales entre la iniciativa y los involucrados muestran diferencias entre los tipos definidos. Las iniciativas que buscan la mejora sistémica (Tipo 6.1) contemplan una mayor cantidad de involucrados, sin perjuicio de que, especialmente en el caso del accountability (Tipo 6.2), el vínculo es de tipo impersonal, en el que los establecimientos deben dar cuenta del cumplimiento de los requerimientos establecidos. Cercano a lo anterior, las iniciativas de incentivo financiero (Tipo 5) y las de regulación de procesos y prácticas (Tipo 4) mantienen una relación puntual o centrada en la entrega de la prestación, o bien en la supervigilancia del cumplimiento de requisitos y procesos.

Por su parte, en las iniciativas de desarrollo de capacidades (Tipo 1), generación de condiciones y apoyos para los estudiantes 
(Tipo 2), y entrega de apoyos para la práctica pedagógica (Tipo 3) se incluye un actor adicional —el intermediario o ejecutor-, responsable de implementar los procesos definidos en los establecimientos o con los actores de las comunidades. En estos casos puede existir una relación que tenga mayor permanencia y más instancias de vinculación entre las partes.

\section{La siguiente figura representa la variedad de elementos} identificados en el diseño de las iniciativas analizadas.

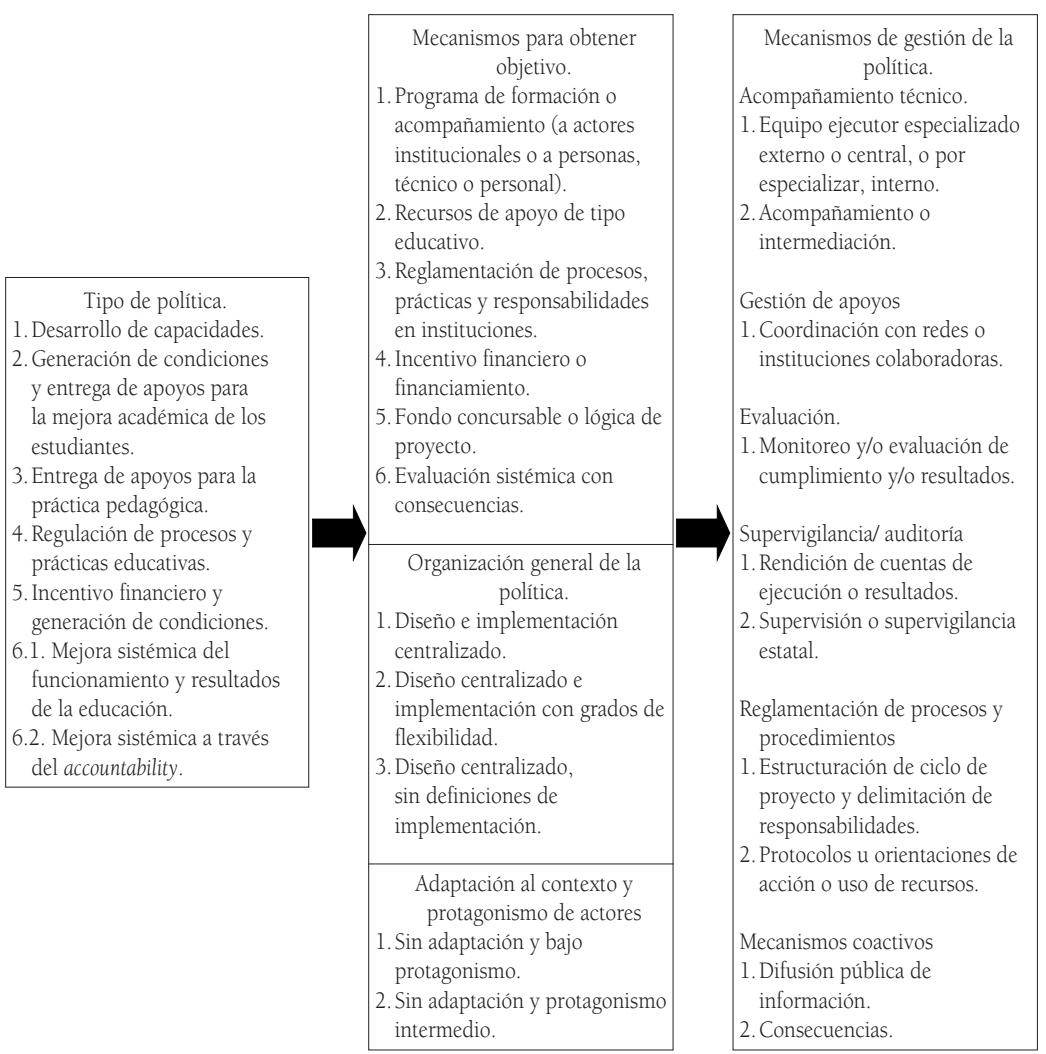

Figura 1.

Características del diseño de las iniciativas.

Fuente: Elaboración propia ${ }^{7}$.

7 Es importante aclarar que este esquema presenta la diversidad de elementos identificados en el diseño de las iniciativas. Esto implica que, dentro de cada ámbito, una iniciativa puede contemplar una o más de las alternativas identificadas. 
Fortalezas y debilidades en la la implementación de las iniciativas

En este apartado se analizan las principales características presentadas en los informes sobre la implementación de las iniciativas, considerando sus fortalezas y debilidades. Estas se han dividido entre: a) aquellos aspectos del diseño que afectan la implementación, y b) fenómenos identificados en la implementación propiamente tales.

En cuanto al primer grupo de fortalezas y debilidades, los contenidos de las iniciativas son, por lo general, valorados en cuanto a su relevancia y aporte al sistema. A ellos, en el caso de las iniciativas cercanas al polo de desarrollo de capacidades (tipos 1 y 2), se agregan las formas de trabajo con las que operan y el vínculo que se establece entre los implementadores y los actores educativos, basados en la cercanía, la flexibilidad y la colaboración.

Por su parte, las políticas orientadas a la mejora sistémica (tipos 6.1 y 6.2) son destacadas en cuanto a su diseño por su capacidad para movilizar a los actores, o por la integralidad o diversidad de elementos que consideran para el logro de sus propósitos.

Respecto de las debilidades del diseño que se evidencian en la implementación, un elemento emerge de manera transversal como deficitario, salvo en el caso de las políticas de mejora sistémica a través de accountability (Tipo 6.2): la no existencia de sistemas de monitoreo, acompañamiento y evaluación. Los reportes verifican una permanente debilidad al respecto, lo que genera dificultades para la toma de decisiones, la corrección de prácticas, el establecimiento de vínculos entre las partes y la estabilidad y proyección de la intervención.

Otra dificultad que se identifica en políticas de diferente tipo es la inexistencia de equipos o recursos suficientes para la implementación de la iniciativa.

Una situación especial se identifica en aquellas iniciativas orientadas a la regulación de procesos y prácticas (Tipo 4), la de incentivo financiero (Tipo 5) y de mejora sistémica (tipos 6.1 y 6.2). En estos casos, las debilidades de diseño que afectan en la 
implementación están relacionadas con la falta de poder, de parte de los incentivos, para propiciar efectivamente la ejecución o cambio de prácticas que buscan modificar. A esto, en el caso de las iniciativas del Tipo 4, se suma la identificación de otras regulaciones que se presentan en tensión o en contradicción, lo que dificulta su relevancia e implementación.

En relación con las fortalezas propiamente tales identificadas en la implementación, se vuelve a identificar diferencias en relación con el tipo de iniciativa. En las que fueron clasificadas cercanas al polo de desarrollo de capacidades (tipos 1 y 2 ) se identifican fortalezas asociadas a las características del vínculo y la forma de trabajo que conlleva, tales como la cercanía entre las partes o características profesionales de los implementadores, aspectos que generan como consecuencia mayores respuestas positivas y adhesión por parte de las comunidades educativas.

Las políticas que generan incentivos o intervienen sistémicamente destacan en la implementación cuando sus dispositivos son eficaces, es decir, cuando las comunidades se alinean y responden a sus lineamientos.

Respecto de las debilidades identificadas en la implementación, los diferentes tipos de iniciativas comparten de manera transversal el que en los territorios no se disponga o no se haya desarrollado las capacidades o condiciones necesarias para la implementación de las iniciativas y que, al mismo tiempo, la iniciativa no contempla su desarrollo. Este aspecto aparece de forma recurrente en los distintos informes.

Además, las capacidades disponibles también pueden ser débiles desde el punto de vista de los implementadores, expresadas en competencias y en capacidades de gestión, y también cuando en las iniciativas interviene el sostenedor, considerando problemáticas similares o de las condiciones que ofrece para la implementación. A ellas se suman dificultades de comunicación o difusión, así como de una implementación insuficiente de los mecanismos planificados, particularmente en las iniciativas orientadas a la regulación de procesos y prácticas y a la mejora sistémica. 
Las figuras 2 y 3 sintetizan las principales fortalezas y debilidades de la implementación de las iniciativas analizadas, según su tipo.

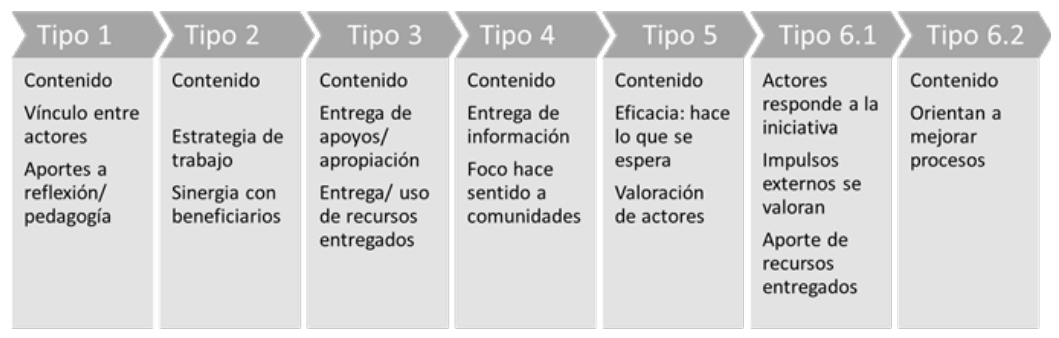

Figura 2.

Fortalezas de la implementación de las iniciativas, según tipo (fortalezas más frecuentes) Fuente: Elaboración propia.

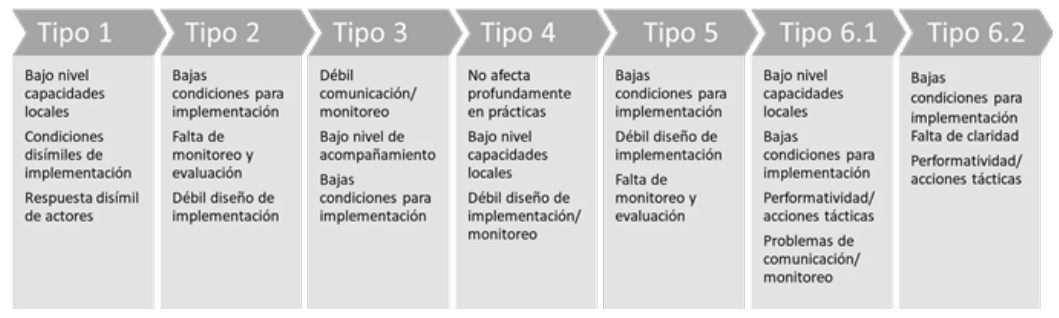

Figura 3.

Debilidades de la implementación de iniciativas, según tipo (debilidades más frecuentes)

Fuente: Elaboración propia.

Interpretaciones, adaptaciones y respuestas a las iniciativas Un aspecto que se identificó con claridad en los reportes de implementación de iniciativas fue la diversidad de interpretaciones respecto de ellas y, llamativamente, las fuentes de las que provienen estas diferencias.

Un primer aspecto que se rescata, principalmente de los tipos de iniciativas más cercanas al desarrollo de capacidades y tipos cercanos (tipos 1, 2 y 3), es que la llegada o interacción directa entre implementadores y usuarios favorece un mayor alineamiento de interpretaciones y de perspectivas con relación a la iniciativa, aspecto que también ayuda a una mejor valoración de esta. 
Esto no quita, sin embargo, que existan brechas de interpretación entre el Estado y los usuarios respecto del uso esperado de determinadas iniciativas, lo que se vislumbra particularmente en el caso de la entrega de recursos educativos, vinculado en los reportes con la existencia de recursos o capacidades para su uso en el nivel local.

La incorporación de otros actores u organismos intermediarios complejiza el alineamiento de interpretaciones, aun cuando la iniciativa tenga espacios de interacción o llegada directa. Así, en algunos casos, los intermediarios conducen la implementación con determinados énfasis o lógicas que no siempre son las esperadas, y que también afectan las capacidades existentes para poder conducir estos procesos. Este fenómeno no se identificó únicamente en las iniciativas de desarrollo de capacidades (Tipo 1), sino también en las de incentivo financiero (Tipo 5).

Otro fenómeno que se distinguió es una mayor distancia de interpretación entre las partes, que tendió a ocurrir cuando habían menores instancias de interacción entre la iniciativa y las comunidades o actores educativos, como es el caso de las iniciativas de regulación de procesos y prácticas (Tipo 4) o las de mejora sistémica (tipos 6.1 y 6.2). Se identificó que los usuarios construyen ciertas visiones respecto de la iniciativa y operan autónomamente basándose en ellas, considerando visiones incompletas o incluso erradas al respecto, o tomando posición respecto de las iniciativas con base en convicciones previas o análisis de costo-beneficio en relación con su incorporación.

Finalmente, se identificó que los equipos o involucrados en la ejecución también interpretan las iniciativas. En iniciativas de diferente tipo se observaron los siguientes fenómenos: variaciones sobre prioridades para la implementación al interior de las reparticiones estatales o entre diferentes unidades u organismos; privilegiar formas de ejecución menos dificultosas, pero no necesariamente más efectivas; no modificar procedimientos o acciones, pese a la existencia de evidencia de errores o problemas. 
La figura 4 da cuenta de las principales interpretaciones que se dieron en las iniciativas analizadas, considerando sus diferentes tipos.

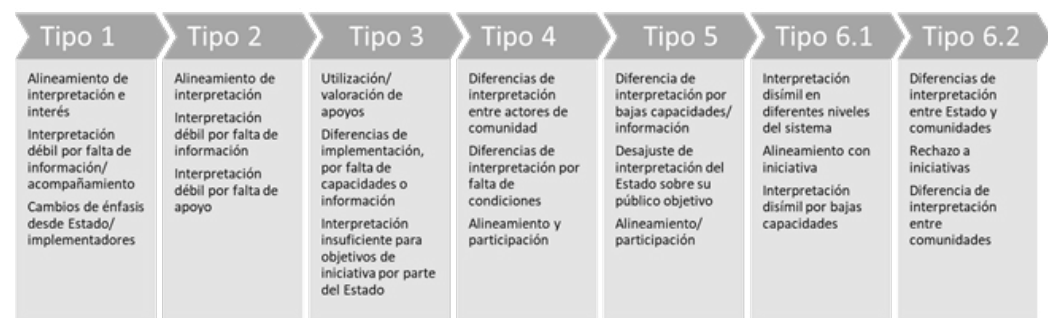

Figura 4.

Principales interpretaciones identificadas en implementación de iniciativas, según tipo Fuente: Elaboración propia.

Se reconocieron, además, diferentes adaptaciones a las iniciativas durante su implementación. Un factor fundamental que explicó estas adaptaciones en diferentes tipos de iniciativa fueron las condiciones y/o capacidades desarrolladas localmente para su implementación. Cuando estas se encuentran en un menor nivel de desarrollo, dificultan o limitan la implementación.

En las iniciativas en las que hay menor espacio de interacción entre el Estado y las comunidades (tipos 4, 5, 6.1 y 6.2), se identificaron adaptaciones cuando los actores buscan dar respuesta a los requerimientos de la iniciativa desde su propia interpretación, o intentando "llenar los vacíos" de procedimientos o acciones que no fueron definidos desde el nivel central.

También se identifican otras adaptaciones por parte del propio Estado, las que se vislumbran con mayor relevancia en los tipos más verticales o con menor interacción. Allí se observan ejemplos de cambios recurrentes desde el nivel central, tales como modificaciones de mecanismos de rendición de cuentas, de plataformas de gestión, o contenidos de proyectos o planes a entregar a la institucionalidad educativa.

Finalmente, las iniciativas con menor grado de adaptación fueron las que tenían una lógica más programática, en que había 
protocolos o procedimientos específicos definidos desde el nivel central, a los que los actores debían ajustarse.

La figura 5 describe las adaptaciones identificadas en la implementación de los diferentes tipos de iniciativa.

\begin{tabular}{|c|c|c|c|c|c|c|}
\hline Tipo & Tipo 2 & Tipo 3 & Tipo 4 & Tіро 5 & Tip & o 6.2 \\
\hline $\begin{array}{l}\text { Priorización de } \\
\text { componentes } \\
\text { por sobre otros } \\
\text { Implementación } \\
\text { deficiente, por } \\
\text { falta de } \\
\text { condiciones, o } \\
\text { bajo estímulo }\end{array}$ & $\begin{array}{l}\text { Implementación } \\
\text { de procesos sin } \\
\text { suficiente } \\
\text { planificación } \\
\text { Implementación } \\
\text { de cambios, sin } \\
\text { suficiente } \\
\text { capacidad } \\
\text { Cambios } \\
\text { consecutivos } \\
\text { desde los } \\
\text { ejecutores }\end{array}$ & $\begin{array}{l}\text { Implementación } \\
\text { de cambios, sin } \\
\text { suficiente } \\
\text { capacidad } \\
\text { Implementación } \\
\text { deficiente, por } \\
\text { falta de } \\
\text { condiciones, o } \\
\text { bajo estímulo } \\
\text { Implementación } \\
\text { de procesos sin } \\
\text { suficiente } \\
\text { planificación }\end{array}$ & $\begin{array}{l}\text { Implementación } \\
\text { de cambios, sin } \\
\text { suficiente } \\
\text { capacidad. } \\
\text { Implementación } \\
\text { deficiente, por } \\
\text { falta de } \\
\text { condiciones, o } \\
\text { bajo estímulo } \\
\text { Adaptación de } \\
\text { trabajo para } \\
\text { calzar con } \\
\text { requerimientos }\end{array}$ & $\begin{array}{l}\text { Implementación } \\
\text { de procesos sin } \\
\text { suficiente } \\
\text { planificación } \\
\text { Implementación } \\
\text { deficiente, por } \\
\text { falta de } \\
\text { condiciones, o } \\
\text { bajo estímulo } \\
\text { Implementación } \\
\text { de cambios, sin } \\
\text { suficiente } \\
\text { capacidad }\end{array}$ & $\begin{array}{l}\text { Implementación } \\
\text { deficiente, por } \\
\text { falta de } \\
\text { condiciones, o } \\
\text { bajo estímulo } \\
\text { Adaptación de } \\
\text { trabajo para } \\
\text { calzar con } \\
\text { requerimientos } \\
\text { Cambios } \\
\text { consecutivos, } \\
\text { desde los } \\
\text { ejecutores } \\
\text { (Estado o } \\
\text { intermediarios). }\end{array}$ & $\begin{array}{l}\text { Adaptación de } \\
\text { trabajo para } \\
\text { calzar con } \\
\text { requerimientos } \\
\text { Decisión de no } \\
\text { responder a la } \\
\text { política } \\
\text { Implementación } \\
\text { de cambios, sin } \\
\text { suficiente } \\
\text { capacidad }\end{array}$ \\
\hline
\end{tabular}

Figura 5.

Principales adaptaciones en la implementación de iniciativas, según tipo

Fuente: Elaboración propia.

En relación con la respuesta de los actores e instituciones a las iniciativas, se identifican varios fenómenos de forma transversal, manteniendo la tónica de que los involucrados buscan permanentemente responder a los requerimientos o invitación realizados centralizadamente por la iniciativa. La forma de respuesta varía en función de dos factores, principalmente.

El primero correspondió a la existencia de condiciones o capacidades para su ejecución en el nivel local, ocurriendo que, cuando ellas estaban disponibles, era más factible alinearse y sacar provecho de los recursos proporcionados. En el segundo, se prioriza, por una parte, una respuesta diferente a la esperada por la iniciativa, sobre la base de concepciones previas respecto del problema que se busca abordar, y, por otra, se cumplen obligaciones mínimas requeridas por la iniciativa, pero sin colaborar o participar de la idea que la iniciativa propone. 
La figura 6 describe las principales respuestas a las iniciativas que se identificaron, considerando su tipo.

\begin{tabular}{|c|c|c|c|c|c|c|}
\hline Tipo 1 & Tipo 2 & Tipo 3 & Tipo & Tipo & 5 & 6.2 \\
\hline $\begin{array}{l}\text { Mediada por } \\
\text { choque con } \\
\text { visiones previas } \\
\text { Mediada por } \\
\text { grado de } \\
\text { flexibilidad y } \\
\text { acompañamiento } \\
\text { de iniciativa } \\
\text { Mediada por } \\
\text { afinidad, } \\
\text { condiciones o } \\
\text { capacidades }\end{array}$ & $\begin{array}{l}\text { Mediada por } \\
\text { afinidad, } \\
\text { condiciones o } \\
\text { capacidades } \\
\text { Mediada por } \\
\text { alineamiento con } \\
\text { requerimientos }\end{array}$ & $\begin{array}{l}\text { Mediada por } \\
\text { afinidad, } \\
\text { condiciones o } \\
\text { capacidades } \\
\text { Mediada por } \\
\text { grado de } \\
\text { flexibilidad y } \\
\text { acompañamiento } \\
\text { de iniciativa } \\
\text { Mediada por } \\
\text { nivel de detalle } \\
\text { de la } \\
\text { implementación }\end{array}$ & $\begin{array}{l}\text { Mediada por } \\
\text { afinidad, } \\
\text { condiciones o } \\
\text { capacidades } \\
\text { Mediada por tipo } \\
\text { de incentivos de } \\
\text { iniciativa } \\
\text { Mediada por } \\
\text { alineamiento con } \\
\text { requerimientos }\end{array}$ & $\begin{array}{l}\text { Mediada por } \\
\text { nivel de detalle } \\
\text { de la } \\
\text { implementación } \\
\text { Mediada por } \\
\text { afinidad, } \\
\text { condiciones o } \\
\text { capacidades }\end{array}$ & $\begin{array}{l}\text { Mediada por } \\
\text { afinidad, } \\
\text { condiciones o } \\
\text { capacidades } \\
\text { Mediada por } \\
\text { choque con } \\
\text { visiones previas } \\
\text { Mediada por tipo } \\
\text { de incentivos de } \\
\text { iniciativa }\end{array}$ & $\begin{array}{l}\text { Mediada por } \\
\text { afinidad, } \\
\text { condiciones o } \\
\text { capacidades } \\
\text { Mediada por tipo } \\
\text { de incentivos de } \\
\text { iniciativa } \\
\text { Mediada por } \\
\text { choque con } \\
\text { visiones previas }\end{array}$ \\
\hline
\end{tabular}

Figura 6.

Tipos de respuestas a las iniciativas durante su implementación, según tipo

Fuente: Elaboración propia.

Centralización o flexibilidad de las iniciativas durante la implementación

Un elemento adicional que se observó en la revisión de los reportes, fue la posibilidad o capacidad de las iniciativas de adaptarse en función del contexto en que se implementan, distinguiéndose con claridad que las iniciativas se ejecutan de forma centralizada. Dentro de ese marco, en las iniciativas de generación de condiciones y apoyos a los estudiantes (Tipo 2), de provisión de recursos (Tipo 3) y normativa (Tipo 4), y las que buscan la mejora sistémica (tipos 6.1 y 6.2), se observa además el fenómeno de que no necesariamente la implementación centralizada implica una definición detallada de los procesos y procedimientos que se requieren para su implementación. Eso implica que las iniciativas se mantienen bajo un aspecto de ejecución autónoma en el nivel local, cuando en realidad se carece de definiciones centrales para su implementación. Esta debilidad debe observarse relacionada con la falta de instancias de vínculo y retroalimentación entre las partes y con la de variedad de adaptaciones y respuestas, lo que interroga en qué medida el Estado da un lugar central al conocimiento de los resultados de sus iniciativas, o si le basta con la ejecución de las acciones planificadas en ellas.

Las iniciativas más próximas al polo de desarrollo de capacidades (tipos 1 y 2 ) muestran ciertos grados de flexibilidad, 
lo que se ve favorecido por el contacto directo entre ejecutores y usuarios, quienes, en algunos casos, pueden hacerse escuchar o escoger elementos dentro de un abanico de oportunidades.

Las iniciativas de entrega de incentivos (Tipo 5) se escapan de los patrones descritos, puesto que su diseño es centralizado, pero a la vez altamente estructurado.

\section{Discusión}

Luego de haber realizado un análisis descriptivo de las principales características de implementación de las iniciativas consideradas para este estudio, en esta sección se presentará un discusión de los resultados, lo que dará paso a la presentación de una propuesta de modelo que permita reconocer los principales factores que intervienen en la implementación de las iniciativas estatales, afectando su aporte al mejoramiento educativo.

Un primer aspecto para comprender la implementación de las iniciativas es el centralismo y verticalidad con el que se desarrolla este proceso, y las escasas diferencias que se asumen desde el diseño en función del contexto en que se ejecutan.

Junto con lo anterior, los resultados muestran que el diseño tiene implicancias en la implementación. Esto significa que, si se considera el continuo que se despliega entre los tipos de iniciativa, desde el desarrollo de capacidades de actores o instituciones hasta la regulación o definición de requerimientos para estos mismos destinatarios, existirán diferencias en la implementación, aun cuando, desde el diseño, se considere la generación de condiciones para la puesta en práctica de la iniciativa.

En segundo lugar, desde el análisis de la implementación propiamente tal, en los resultados se identifican cuatro claves relevantes que debieran estar adecuadamente desarrolladas en las iniciativas que buscan la mejora. La primera clave, tal vez la más importante de todo el proceso, corresponde a la generación, apoyo o aseguramiento del desarrollo de capacidades necesarias para la implementación de 
las iniciativas y su sustentabilidad, lo que emerge como una de las grandes carencias identificadas en los reportes analizados. Estas capacidades — profesionales y organizacionales - competen tanto al Estado central como al nivel intermedio y las propias organizaciones escolares, y sin ellas no es dable esperar que las iniciativas puedan sostenerse y lograr sus propósitos fundamentales.

Dentro de este marco general, se observó que las iniciativas más ambiciosas en cuanto al impacto esperado (mejora sistémica, regulación) fueron justamente aquellas que disponían de menores instancias o mecanismos para desarrollar o fortalecer capacidades en el nivel local, que eran indispensables para el logro de sus objetivos. El efecto de este problema es muy relevante, debido a que, en su "bajada", no hay responsables evidentes del desarrollo de estas capacidades, lo que se convierte en un alto riesgo de desigualdad: los actores u organismos con mayores condiciones y capacidades podrían utilizar y aprovechar de mejor forma los recursos del Estado que quienes no han podido desarrollar estas capacidades, o donde no existen suficientes condiciones para la implementación Como pudo observarse, otra de las grandes debilidades identificadas fueron las deficiencias o desigualdades en las condiciones necesarias para la ejecución de las iniciativas.

La segunda clave es la eficacia, es decir la capacidad de realizar lo que está previamente planificado. Se comprende como requisito que la iniciativa debe contar con un adecuado diseño, tanto de su estructura como de su implementación, en el que se definan y detallen los procesos, criterios y procedimientos necesarios de desarrollar, y que, luego, estos efectivamente se ejecuten. Estos atributos no estuvieron siempre presentes en los informes analizados.

Una tercera clave dice relación con los vínculos que se establecen entre el ejecutor y los usuarios o participantes de la iniciativa. Particularmente, las relaciones bidireccionales son aquellas que favorecen en mejor medida la mejora, lo que supone la capacidad de retroalimentar mutuamente a las partes y que ello favorezca el ajuste del trabajo a realizar en función de los objetivos a alcanzar. Esto implica la existencia de procesos específicos de monitoreo y 
evaluación —que también emergen como ausentes en una gran cantidad de iniciativas- y de cierta disposición de parte del Estado por comprender la implementación como algo más que la entrega de un bien o un servicio.

Una parte importante de las iniciativas analizadas se muestran atrapadas en una lógica burocrática de operación, y parecen conformarse con la ejecución de acciones planificadas como parámetro de éxito de su implementación, en vez de la búsqueda de evidencia sobre el efecto que tienen sobre la mejora.

Finalmente, la cuarta clave alude a la coherencia interna entre iniciativas y la capacidad de éstas de complementarse o, al menos, de no competir entre sí a la hora de intentar abordar la resolución de determinado problema público. Como se evidenció en los resultados, esto puede tener implicancias muy relevantes en los logros que se alcancen.

Del análisis surge que hay otros tres procesos que ocurren en la implementación y que son necesarios para comprender y evaluar el aporte de una iniciativa al mejoramiento educativo. El primero es la interpretación que se haga de la iniciativa, tanto de parte de los actores y organismos en el nivel local, como del propio Estado y de potenciales instituciones intermediarias. Desde una perspectiva sintética, podría indicarse que la convergencia en las interpretaciones respecto de las iniciativas entre los diferentes involucrados podría aportar a una implementación orientada a la mejora.

Mediante la interpretación o reinterpretación, los actores educativos pueden, de manera racional, definir y priorizar el uso que dan a las iniciativas e intentar aprovecharlas en función de sus propias capacidades, alineándose o no con las definiciones de la iniciativa. Sin embargo, tal como se mencionó, si estas capacidades no están desarrolladas de acuerdo con lo esperado, igualmente las iniciativas no podrán utilizarse como se espera, aun cuando exista alineamiento con la propuesta de la iniciativa. 
Como consecuencia de la interpretación, se puede comprender la respuesta que los usuarios de las iniciativas tienen respecto de éstas (segundo proceso). Es importante comprender que la respuesta se entiende como una reacción mediada por las claves mencionadas y el proceso de interpretación. Por esta razón, se podría indicar que las respuestas se mueven entre un polo mayormente mediado por la iniciativa (o por las definiciones del Estado) y otro mayormente mediado, de manera interna, por el actor u organismo correspondiente. Estos polos se ven afectados además por el grado de desarrollo de capacidades y condiciones para la implementación.

En línea con lo señalado, las adaptaciones a la iniciativa (tercer proceso) también serán consecuencia o estarán relacionadas con los aspectos que se han descrito, y estarán también más mediadas por la política o de forma interna. Como se observó, una parte importante de las adaptaciones se dan debido a carencias de la iniciativa, mientras otras ocurren como consecuencia de las interpretaciones y las respuestas que se dan a ella. Todas estas posibilidades abren diferentes caminos en torno al aporte a la mejora. Por su parte, se distinguieron pocas iniciativas en las que las adaptaciones eran producto de acuerdos entre las partes en el nivel local. Cuando ello ocurrió, las iniciativas eran mayormente valoradas y contaban con mayor adherencia de parte de las comunidades.

La figura 7 organiza en un modelo los componentes mencionados y su incidencia en el proceso de implementación orientado al mejoramiento educativo. Su conformación constó de dos etapas. La primera consistió en una primera versión, elaborada a partir del análisis descriptivo presentado en la sección de resultados de este artículo. La segunda se realizó a partir de la sistematización de observaciones y sugerencias surgidas en las entrevistas a representantes de equipos de investigación. Con estos antecedentes se terminó de diseñar el modelo que se presenta a continuación.

El modelo, descrito con un esquema "desde arriba hacia abajo" — con base en los resultados presentados_- cuenta con cinco grandes dimensiones de factores que afectan en la implementación: a) diseño (foco y características de la iniciativa); b) puesta en práctica (procesos 
implicados en la ejecución de la iniciativa); c) interpretación (análisis de los involucrados respecto de la iniciativa y sus implicancias); d) respuesta (reacción de los involucrados respecto de la iniciativa), y e) adaptación (cambios ejecutados al diseño de la iniciativa).

Cada una de estas dimensiones está compuesta por factores que afectan la implementación y que han sido anteriormente mencionadas. Por su parte, el desarrollo de capacidades y disponibilidad de condiciones emergen como factores que afectan transversalmente la implementación, considerando las diferentes dimensiones mencionadas.
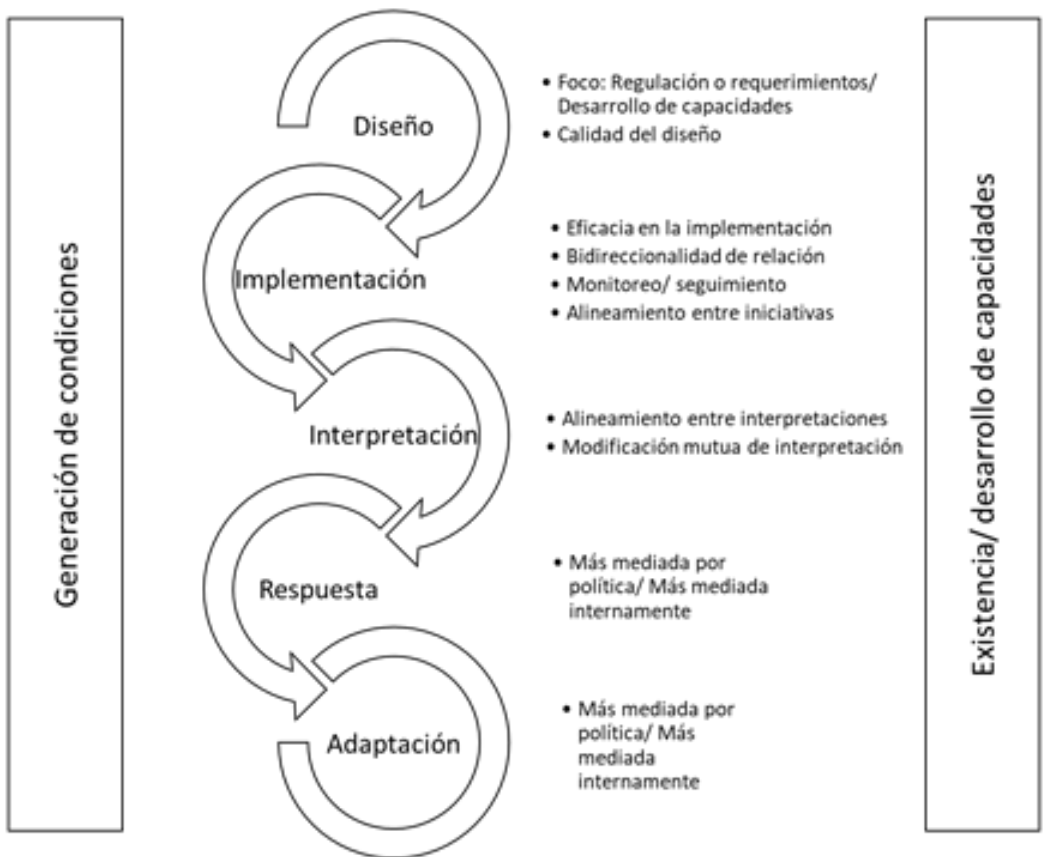

Figura 7.

Modelo para el análisis de la implementación de iniciativas orientadas al mejoramiento educativo

Fuente: Elaboración propia.

Dentro del análisis realizado, parece relevante realizar una última profundización respecto de las iniciativas más ambiciosas: las que buscan la mejora sistémica. Estas, además, representan las expectativas 
del Sistema de Aseguramiento de la Calidad de la Educación. La lógica vertical, la prioridad de definir regulaciones y requerimientos, y la menor presencia de mecanismos de acompañamiento y desarrollo de capacidades en estas iniciativas, se pueden interpretar — desde los resultados presentados - como un énfasis performativo de parte de las políticas educativas. Es a través de mecanismos de control e incentivo, más que de diálogo y acompañamiento, que el Estado parece buscar permear las prioridades, lógicas de acción y procesos al interior de los establecimientos educacionales. Las iniciativas de mayor envergadura, que evidenciaron un mayor aporte a la mejora y que se posicionan en esta lógica, deben realizar un esfuerzo adicional para lograr cambios, acompañando estos atributos con la disposición de recursos materiales y financieros, y mecanismos de comunicación y participación de múltiples actores y organismos en las diferentes capas del sistema.

Es decir, existe evidencia que permite señalar que este énfasis puede tener efectos sobre la mejora, pero considerando un resultado que se entenderá como la agregación de un conjunto de esfuerzos y no solo de una iniciativa. Sin embargo, en los casos analizados hay más ejemplos en los que el Estado parece conformarse con una visión burocrática de los resultados esperados, esperando que los actores educativos den cuenta del cumplimiento de ciertas responsabilidades y requerimientos, pero sin que ello necesariamente implique una real mejora. De esta forma, la lógica de implementación de las iniciativas de mayor envergadura no se basa en el desarrollo de capacidades, ni tampoco en los resultados, sino en una burocracia performativa.

\section{Referencias}

Anderson, S., Mascall, B., Stiegelbauer, S. \& Park, J. (2012). No one way: differentiating school district leadership and support for school improvement. Journal of Educational Change, 13, 403- 430.

Aziz, C. (2018). Evolución e implementación de las politicas educativas en Chile. Nota Técnica No2. Centro de Liderazgo Líderes Educativos. Recuperado de: https://www.lidereseducativos.cl/wp-content/uploads/2018/06/ NT2_L6_C.A_Evolucio\%CC\%81n-e-implementacio\%CC\%81n-delas-poli\%CC\%81ticas-educativas-en-Chile.pdf 
Bardach, E. (1976). The implementation game: what happens after a bill becomes a law. Cambridge, London: The MIT Press.

Bardin, L. (1996) Análisis de contenido. Madrid: Akal Ediciones.

Barrett, S. M. (2004). Implementation studies: Time for a revival? Personal reflections on 20 years of implementation studies. Public Administration, 82(2), 249-262. DOI: 10.1111/j.0033-3298.2004.00393.x.

Bellei, C., Valenzuela, J., Vanni, X. y Contreras, D. (2014). Lo aprendí en la escuela. ¿Cómo se logran procesos de mejoramiento escolar? Santiago de Chile: Universidad de Chile-UNICEF.

Botella, J. y Zamora, Á. (2017). El meta-análisis: una metodología para la investigación en educación. Educación XX1, 20(2), 17-38.

Bryant, D., Ko \& J. Walker, A. (2018). How Do School Principals in Hong Kong Shape Policy? Leadership and Policy in Schools, 17(3), 345-359. DOI: 10.1080/15700763.2018.1496340.

Cáceres, P (2003). Análisis Cualitativo de Contenido: una alternativa metodológica alcanzable. Psicoperspectivas, 2(1), 53-82.

Coburn, C. (2003). Rethinking scale: Moving beyond numbers to deep and lasting change. Educational Researcher, 32(6), 3-12.

Coburn, C., Hill, H. \& Spillane, J. (2016). Alignment and Accountability in Policy Design and Implementation: The Common Core State Standards and Implementation Research. Educational Researcher, 45(4), 243-251.

Chrispeels, J., Harris, A. (Eds.). (2006). Improving Schools and Educational Systems: International Perspectives. London: Routledge. 2006.

Datnow, A. (2000). Power and Politics in the Adoption of School Reform Models. Educational Evaluation and Policy Analysis, 22(4), 357-374.

Datnow, A. (2005). The Sustainability of Comprehensive School Reform Models in Changing District and State Contexts. Educational Administration Quarterly, 41(1), 121-153.

Datnow, A. \& Park, V. (2009). Conceptualizing policy implementation: largescale reform in an era of complexity. In: G. Sykes \& P. Schneider (Eds.), Handbook of educational policy research. Nueva York: American Educational Research Association.

Day, C. \& Gu, Q. (2018) How Successful Secondary School Principals in England Respond to Policy Reforms: The Influence of Biography. Leadership and Policy in Schools, 17(3), 332-344. DOI: 10.1080/15700763.2018.1496339. 
Dedering, K. \& Muller, S. (2011). School improvement through inspections? First empirical insights from Germany. Journal of Educational Change, 12, 301-322.

De Leon, P. \& Varda, D. M. (2009). Toward a Theory of Collaborative Policy Networks: Identifying Structural Tendencies. Policy Studies Journal, 37(1), 59-74. DOI: 10.1111/j.1541-0072.2008.00295.x

Durlak, J. A. \& Dupré, E. (2008). Implementation Matters: A Review of Research on the Influence of Implementation on Program Outcomes and the Factors Affecting Implementation. American Journal of Community Psychology, 41, 327-350.

Elmore, R. F. (1996). Getting to scale with good educational practice. Harvard Educational Review, 66(1), 1-26.

Elmore, R. F. (2003). Diseño retrospectivo: la investigación de la implementación y las decisiones políticas (G. Bernal, Trans.). En: L. T. Aguilar (Ed.), La implementación de las políticas (pp. 251-280). México: Miguel Ángel Porrúa.

Elmore, R. (2010). Mejorando la escuela desde la sala de clases. (C. Santa Cruz, Trans.). Santiago de Chile: Fundación Chile, Fundación CAP.

Fullan, M. (2002). Los nuevos significados del cambio en la educación. Barcelona: Octaedro.

Fullan, M. (2010). Positive pressure. In: A. Hargreaves, A. Lieberman, M. Fullan \& D. Hopkins (Eds.), Second international handbook of educational change (pp. 119-130). Dordrecht: Springer Science+Business Media.

Fullan, M. (2011). Choosing the wrong drivers for whole system reform. Centre for Strategic Education. Seminar Series Paper, 204.

Fullan, M. \& Rincon-Gallardo, S. (2016). Developing High Quality Public Education in Canadá. In: F. Adamson, B. Astrand \& L. DarlingHammond (Eds.), Global Education Reform. How Privatization and Public Investment Influence Education Outcomes. London: Routledge.

Gallucci, C., Knapp, M., Markholt, A. \& Ort, S. (2006). Standards- based Reform and Small School of Choice. How reform Theories converge in Three Urban Middle Schools. In: A. Harris \& J. Chrispeels (Eds.), Improving schools and educational systems. International perspectives. New York: Routledge.

Gill, B.; Coffee-Borden, B. \& Hallgren, K. (2014). A Conceptual Framework for Data-Driven Decision Making. Recuperado de https://www. mathematica.org/our-publications-and-findings/publications/aconceptual-framework-for-data-driven-decision-making 
Gu, Q., Sammons, P. \& Chen, J. (2018). How Principals of Successful Schools Enact Education Policy: Perceptions and Accounts from Senior and Middle Leaders. Leadership and Policy in Schools, 17(3), 373-390. DOI: 10.1080/15700763.2018.1496344.

Guhn, M. (2009). Insights from successful and unsuccessful implementations of school reforms programs. Journal of Educational Change, 10, 337 363.

Hallinger, P. \& Heck, R. (2011). Exploring the journey of school improvement: classifying and analyzing patterns of change in school improvement processes and learning outcomes, school effectiveness and school improvement. International Journal of Research, Policy and Practice, 22(1), 1-27.

Hargreaves, A. (2010). The fourth way of change: A Hopeful Struggle. In: A. Hargreaves y M. Fullan (Eds.), Change Wars. Bloomington: Solution Tree.

Hargreaves, A. \& Fullan, M. (2012). Professional Capital. Transforming teaching in every school. New York: Teacher College Press; Abingdon: Routledge.

Hargreaves, A. \& O'Connor, M. (2018). Collaborative Professionalism: When Teaching Together Means Learning for All. Corwin Impact Leadership Series.

Harris, A. (2012). Liderazgo y desarrollo de capacidades en la escuela. (C. Santa Cruz, Trans.). Santiago de Chile: Fundación Chile, Fundación CAP.

Harris, A., Jones, M. \& Adams, D. (2016) 'Qualified to Lead? A Comparative, Contextual and Cultural View of Educational Policy Borrowing. Educational Researcher, 58(2), 166- 178. DOI: 10.1080/00131881.2016. http://dx.doi.org/10.1080/00131881.2016.1165412

Hopkins, D. (2008). Hacia una Buena Escuela: Experiencias y Lecciones. (C. Santa Cruz, Trans.). Santiago de Chile: Fundación Chile, Fundación CAP.

Hopkins, D. (2013). Exploding the myths of school reform. Open University Press, McGraw Hill Education, Berkshire.

Hopkins, D. (2017). The Past, Present and Future of School Improvement and System Reform. An expanded version of the William Walker Oration, ACEL National Conference, Sydney, NSW, 6 ${ }^{\text {th }}$ October 2017.

Kit Looi, C. \& Woon Teh, L. (2015). Scaling Educational Innovations. Springer Education Innovation Book Series. Singapore: Springer.

Lester, J., Lochmiller, Ch. \& Gabriel, R. (2017). Discursive Perspectives on Education Policy and Implementation. Palgrave Macmillan. 
Levin, B. (2007). Sustainable, large-scale education renewal. Journal of Educational Change, 8, 323-336.

Levin, B. (2009). Does politics help or hinder education change? Journal of Educational Change, 10, 69-72.

Lochmiller, Ch. \& Hedges, S. (2017). Education Policy Implementation Research: A Call for New Approaches. In: J. Lester, Ch. Lochmiller $\&$ G. Rachael (Eds.), Discursive Perspectives on Education Policy and Implementation. Palgrave MacMillan.

Mourshed, M., Chijioke, C. \& Barber, M. (2010). How the world's most improved school systems keep getting better. In: M. Mourshed, C. Chijioke \& M. Barber (Eds.), How the world's most improved school systems keep getting better. London: McKinsey and Company.

Nilsen, P. (2015). Making sense of implementation theories, models and frameworks. Implementation Science, 10(1), 53-66.

Parsons, W. (2007). Políticas Públicas: una introducción a la teoría y la práctica del análisis de políticas públicas (A. Acevedo, Trans.). Buenos Aires: Facultad Latinoamericana de Ciencias Sociales.

Pülzl, H. T. O. (2007). Implementing public policy. In: F. M. Fischer G. \& M. Sidney (Ed.), Handbook of Public Policy Analysis: Theory, Politics, and Methods (pp. 89-107). New York: Taylor and Francis.

Revuelta, R. (2008). La implementación de políticas públicas. Dikaion, 21(16), 135-156.

Rincón-Gallardo, S. (2014). Innovación pedagógica en gran escala: ¿Lujo o imperativo moral? Didac, 65, 11-18.

Rincon-Gallardo \& S.; Fleish, B. (2016). Bringing effective instructional practice to scale: An introduction. Journal of Educational Change, 17, 379-383.

Sabatier, P. A. M. (2003). La implementación de la política pública: un marco de análisis (G. Bernal, Trans.). En: L. Aguilar (Ed.), La implementación de las políticas (pp. 323-372). México: Miguel Ángel Porrúa.

Sanetti, L. \& Collier-Meek, M. (2015). Data-Driven Delivery of Implementation Supports in a Multi-Tiered Framework: A Pilot Study. Psychology in the Schools, 52(8), 815-828.

Schleicher, A. (2016). Teaching Excellence through Professional Learning and Policy Reform: Lessons from Around the World. International Summit on the Teaching Profession. Paris: OECD Publishing. DOI: http://dx.doi. org/10.1787/9789264252059-en 
Spillane, J., Reiser, B. \& Reimer, T. (2002). Policy implementation and cognition: Reframing and refocusing implementation research. Review of Educational Research, 72(3), 387-431.

Strunk, K., Marsh, J., Hashim, A. \& Bush-Mecenas, S. (2016). Innovation and a Return to the Status Quo: A Mixed- Methods Study of School Reconstitution. Educational Evaluation and Policy Analysis, 38(3), 549577.

Strunk, K., Marsh, A., Bush-Mecenas, S. \& Duque, R. (2016). The Best Laid Plans: An Examination of School Plan Quality and Implementation in a School Improvement Initiative. Educational Administration Quarterly, 52(2) 259-309.

Valles, M. (2000). Técnicas cualitativas de investigación social. Madrid: Editorial Síntesis.

Van Meter, D. (1975). El proceso de implementación de las políticas. Un marco conceptual. En: L. T. Aguilar (Ed.), La implementación de las políticas (pp. 97-146). México: Miguel Ángel Porrúa.

Weiss, C. (1972). Evaluation research. New York: Prentice Hall. 